\title{
Editorial
}

\section{Recent Theory and Applications on Inverse Problems 2014}

\author{
Fatih Yaman, ${ }^{1}$ Valery G. Yakhno, ${ }^{2}$ Caner Özdemir, ${ }^{3}$ Tzu-Yang Yu, ${ }^{4}$ and Roland Potthast ${ }^{5,6}$ \\ ${ }^{1}$ Department of Electrical and Electronics Engineering, Izmir Institute of Technology, Gülbahce-Urla, 35430 Izmir, Turkey \\ ${ }^{2}$ Department of Electrical and Electronics Engineering, Dokuz Eylul University, 35160 Izmir, Turkey \\ ${ }^{3}$ Department of Electrical and Electronics Engineering, Mersin University, 33343 Mersin, Turkey \\ ${ }^{4}$ Department of Civil and Environmental Engineering, University of Massachusetts Lowell, Lowell, MA 01854-2827, USA \\ ${ }^{5}$ Department of Mathematics, University of Reading, Whiteknights, P.O. Box 220, Reading RG6 6AX, UK \\ ${ }^{6}$ German Meteorological Service, Deutscher Wetterdienst Research and Development, Head Division FE 12 (Data Assimilation), \\ Frankfurter Strasse 135, 63067 Offenbach, Germany
}

Correspondence should be addressed to Fatih Yaman; fatihyaman@iyte.edu.tr

Received 25 December 2014; Accepted 25 December 2014

Copyright (C) 2015 Fatih Yaman et al. This is an open access article distributed under the Creative Commons Attribution License, which permits unrestricted use, distribution, and reproduction in any medium, provided the original work is properly cited.

This special issue presents some very interesting recent developments in the area of inverse problems. The scope of the issue covers wide range of disciplines, for example, electromagnetics, acoustics, heat conduction, and image processing, from theory and application point of view. In the following, we give very brief descriptions of the published papers.

M. R. Santos et al. propose the estimation of heat flux at the chip-tool interface using inverse technique. The paper demonstrates an elaborate experimentation. The nonlinear heat diffusion equation is solved via a $3 \mathrm{D}$ numerical code and the temperature distribution is predicted using finite volume elements. For the inverse problem, the function specification method is employed. Heat fluxes at the tool-workpiece interface are estimated using inverse problems methods and experimental temperatures. The results are matched in a fair way with those of the tool-work thermocouple technique for cutting parameters on cutting edge temperature.

C. He et al. present an adaptive TGV-based model for noise removal. The aim of the study is to achieve a balance between edge preservation and region smoothness for image denoising. The variable splitting and the classical augmented Lagrangian method (ALM) are employed in the solution of the proposed method. The authors observe that the proposed algorithm is effective in suppressing staircasing effect and preserving edges in images, and it is superior to some other famed adaptive denoising methods both in quantitative and in qualitative assessment.
$\mathrm{X}$. Zeng proposes a bound alternative direction method which can be considered as an extension of alternating direction method of multipliers for the solution of $\ell_{p}(p \in(0,1))$ minimization problems in image deblurring. The author reports that the experiments on a set of image deblurring problems have shown that the proposed method for the synthesis $\ell_{p}$ formulation is favorably competitive with the state-of-the-art algorithms for the synthesis $\ell_{1}$ formulation.

C. Özdemir et al. present a review paper whose aim is to evaluate and compare the migration algorithms over different focusing methods such that the reader can decide which algorithm to use for a particular application of GPR. In the paper, the brief formulation and the algorithm steps for the hyperbolic summation, the Kirchhoff migration, the backprojection focusing, the phase-shift migration, and the $(\omega-k)$ migration are presented and the simulated and the measured examples that are used for the performance comparison of the presented algorithms are provided.

Y. He et al. investigate the flight maneuvering trajectories evaluation problem. The algorithm differentiation is used to realize the inverse computation of the rotor-flying robot system. The advantages of the proposed algorithms are mentioned as only desired positions and their derivatives and the feasibility of the trajectories (including inner states and inputs) can be evaluated and additionally an accurate pointwise numerical feasibility analysis of planned trajectories becomes possible. 
C. Deng et al. consider a compound regularization based compressive sensing MRI reconstruction model, which exploits the NLTV regularization and wavelet approximate sparsity prior. For the algorithm, the variable splitting and augmented Lagrangian algorithm is applied to solve the compound regularization minimization problem. Authors report that the experiments on test images demonstrate that the proposed method leads to high SNR measure and more importantly preserves the details and edges of MR images.

J. Wang et al. propose a strategy using joint angleDoppler representation basis which can determine a sparse target scenario in spatial domain at the same range for active radar in the forward-looking direction. According to the authors, the presented approach settles the trouble that traditional SAR and DBS techniques cannot provide an image for active radar in the line of sight and needs only singlereceiver channel without any modification on traditional radar hardware. The given strategy shows good performance with different setup about SNR level and target numbers.

A. Palafox et al. pose an acoustic inverse scattering problem in a Bayesian inference perspective and simulate the posterior distribution using Markov chain Monte Carlo (MCMC). For the corresponding direct problem, the classical layer potential approach is employed and the problem was solved in a fast and reliable manner with parallel computing. The authors implemented the effective dimension method via Bayesian model selection where the normalizing constant for each model is approximated using the MCMC output for giving a parametric representation of the solution of the inverse problem.

D. Wang et al. apply the adjoint tidal model based on the theory of inverse problem to investigate the effect of bottom friction coefficient on the tidal simulations. In their work, the Bohai, Yellow, and East China Sea are simulated by assimilating altimeter data. Authors report that the simulated results with new empirical formulas are better than traditional schemes, such as the constant, different constant in different subdomain, and depth-dependent form.

F. Mohebbi and M. Sellier study an optimal shape design problem in aerodynamics. The aim of the inverse problem is finding the optimal shape of an airfoil placed in a potential flow at a given angle of attack should have such that the pressure distribution on its surface matches a desired one. To achieve this aim, a numerical method is investigated to generate a mesh over the airfoil surface and to solve for the flow equation. The authors report in their paper that the proposed sensitivity analysis method reduces the computation cost even for large number of the design variables and confirm accuracy and efficiency of the proposed shape optimization algorithm.

K. T. Iskakov and Zh. O. Oralbekova consider the direct and inverse problems for the geoelectric equations. They have showed that the considered problem is reducible to a system of the Volterra integral equations. A stability estimate has been proved for the solution of this system. As a result, a conditional stability estimate has been obtained for the solution of the considered inverse problem. The application of this conditional stability estimate is discussed.
H.-D. Yeh et al. propose an approach to solve complicated source release problems in groundwater contaminant plumes, containing unknowns of three location coordinates and several irregular release periods and concentrations. The authors employ an ordinal optimization algorithm (OOA), roulette wheel approach, and a source identification algorithm, based on simulated annealing, tabu search, and a three-dimensional groundwater flow and solute transport model. It is demonstrated that the proposed approach works effectively in the problems with different initial guesses of source location and measurement errors and with large suspicious areas and several source release periods and concentrations.

C. Ak and A. Yildiz propose an inverse approach to obtain a relation between applied voltage and displacement of the midpoint of fixed-fixed beam actuator. An inversely designed model and a modified formula which establishes a good approximation of the system with an unsophisticated representation are presented. In this direction, one can calculate required voltage for pull-in limit in a simpler way. On the other hand, the approach, for example, does not take the fringing effect into account for the sake of simplicity. However, employment of artificial optimization techniques is suggested for the improvement of the modified formula.

G. Jin et al. study the inversion of vertical eddy viscosity coefficient (VEVC) with a method based on an isopycnic coordinate internal tidal model in their paper. Numerical experiments are provided to examine the influence factors on the inversion of VEVCs in four aspects: independent point schemes (IPS), topography, the spatial distribution of VEVC, and the optimization methods. The authors found out in their investigations that the limited-memory Broyden-FletcherGoldfarb-Shanno (L-BFGS) method is a more effective method than the gradient descent method (GDM-S) in terms of the inversion of the VEVC. However, it is noted that the GDM-S is more convenient and controllable, so it should not be ignored and should be taken seriously as a choice for the inversion of the VEVC with spatial distribution.

Z. Ruan et al. study a backward problem for a timefractional diffusion equation. This ill-posed problem is formulated as a regularized optimization problem. The authors propose a direct numerical algorithm for solving the stated problem. This algorithm is based on the adoption Tikhonov regularization to overcome ill-posedness. The regularization parameters are selected from computational experiments by the discrepancy principle. The numerical results confirm the robustness of the algorithm.

\section{Acknowledgments}

The guest editors would like to deeply thank all the authors, the reviewers, and the editorial board involved in the preparation of this issue.

Fatih Yaman Valery G. Yakhno Caner Özdemir Tzu-Yang Yu Roland Potthast 


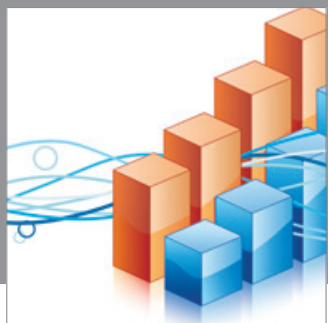

Advances in

Operations Research

mansans

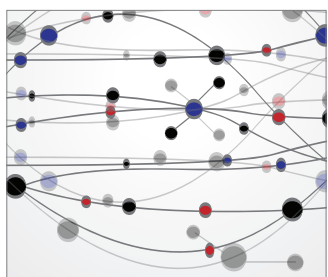

The Scientific World Journal
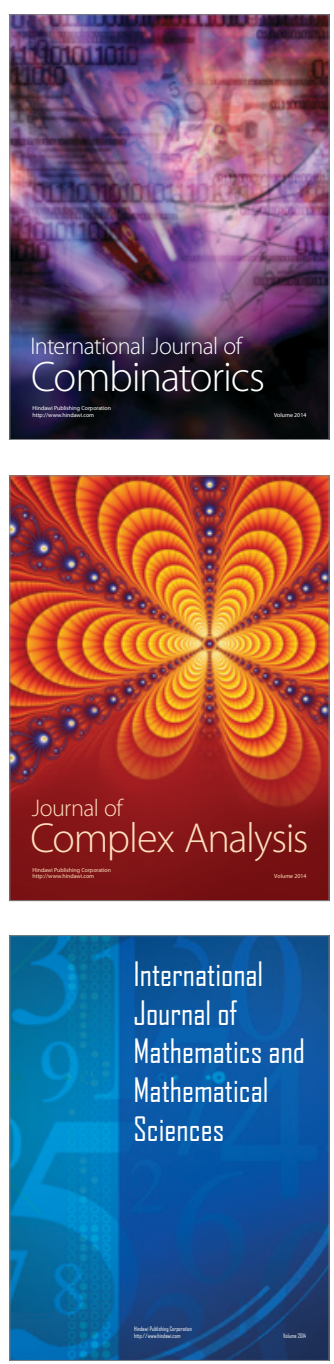
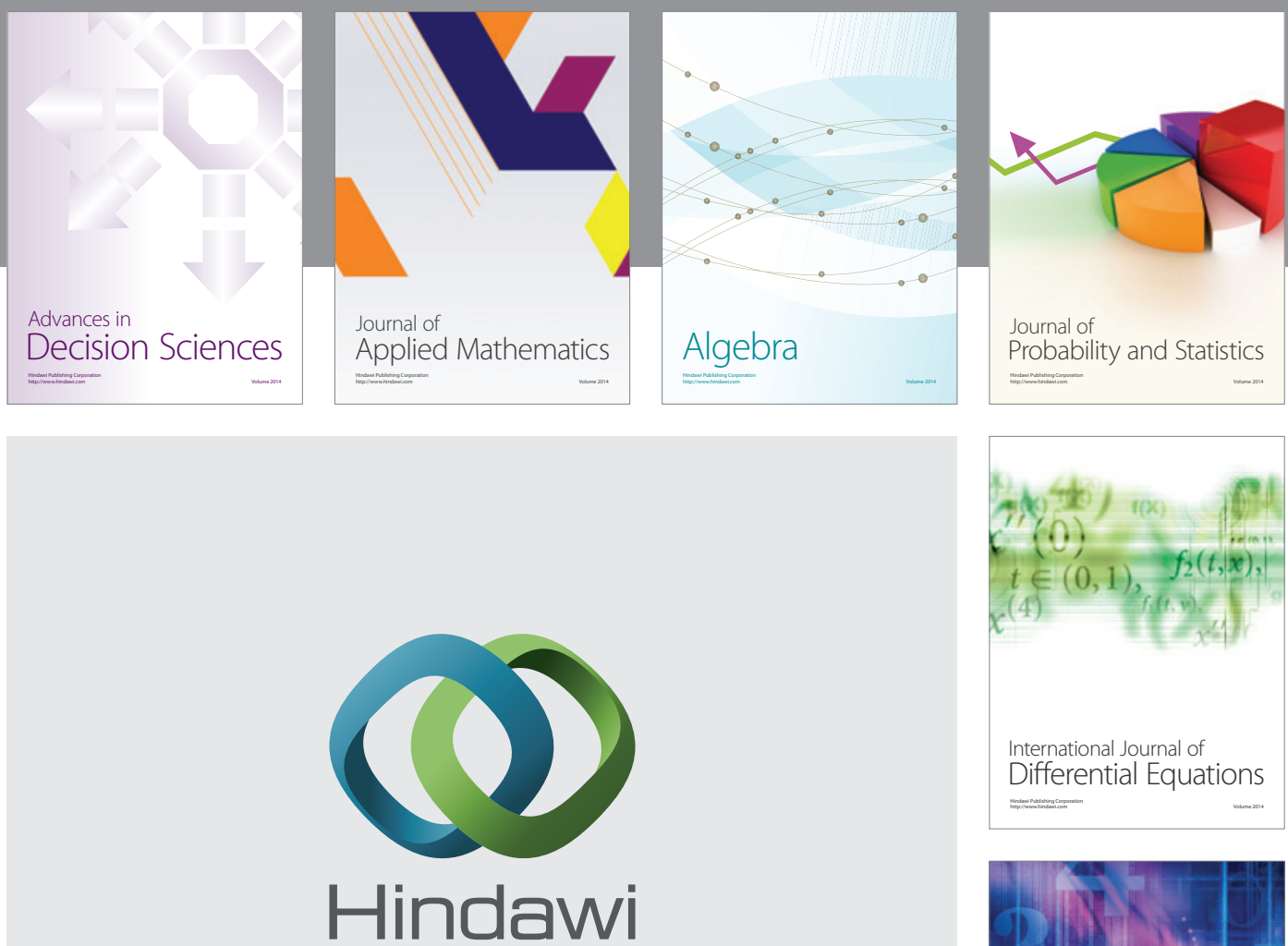

Submit your manuscripts at http://www.hindawi.com
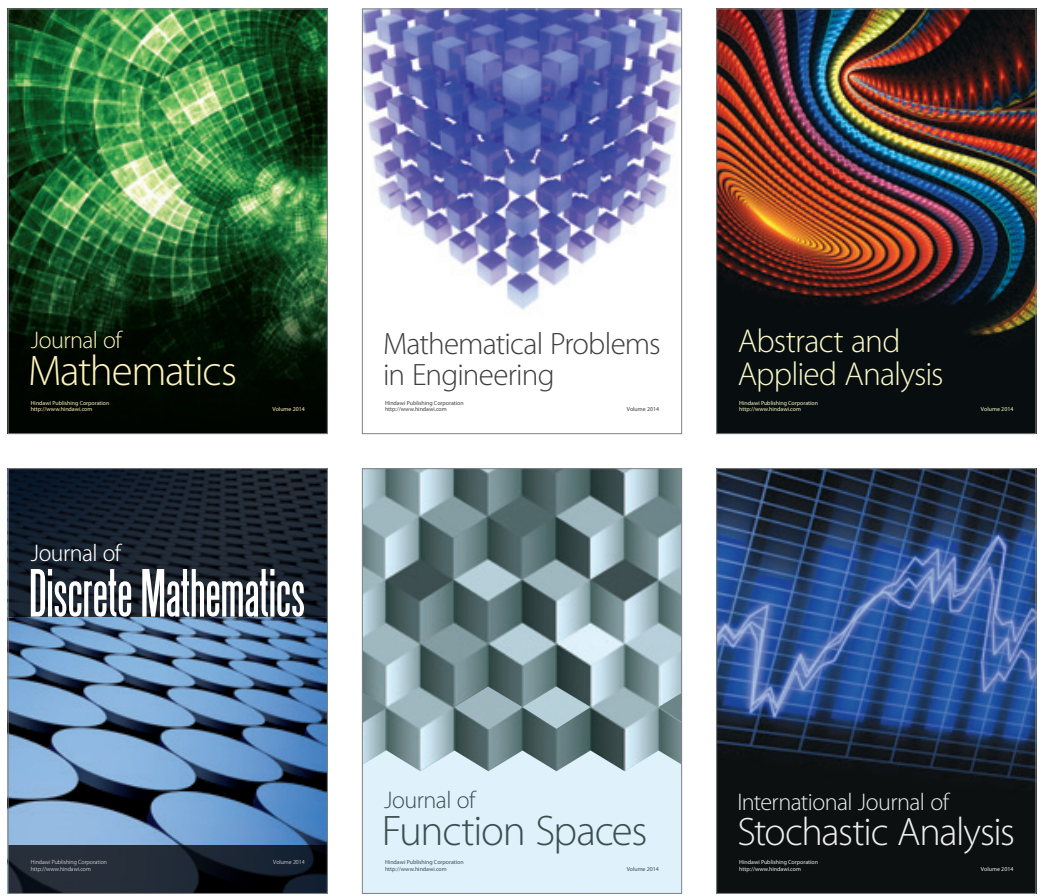

Journal of

Function Spaces

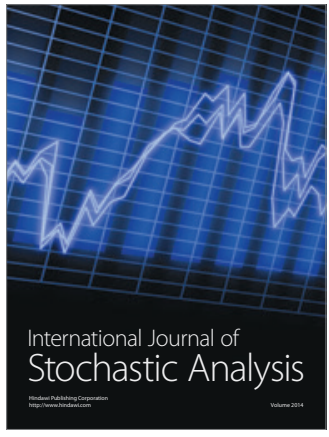

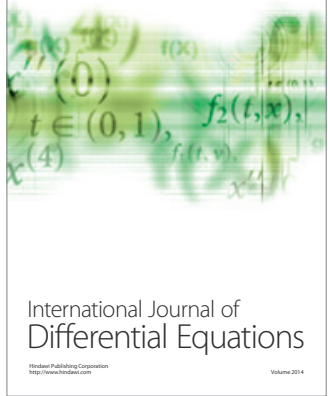
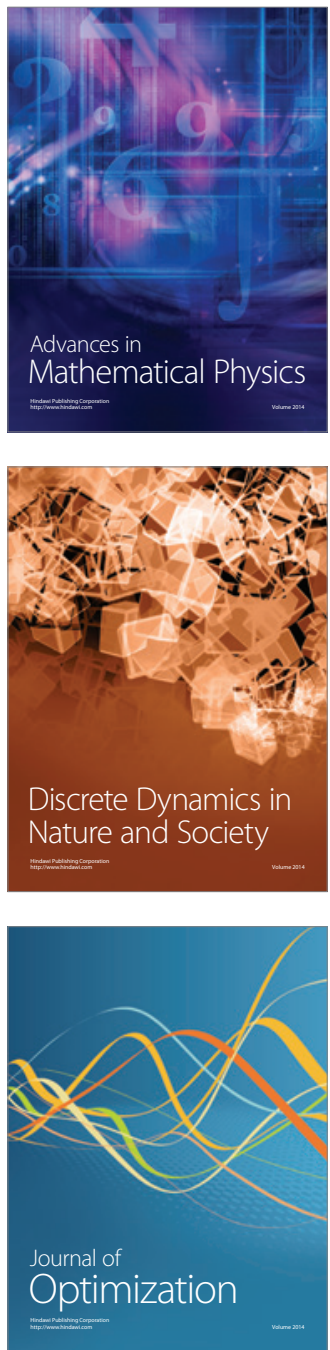